\title{
Tumor Identifier Original Result
}

National Cancer Institute

\section{Source}

National Cancer Institute. Tumor Identifier Original Result. NCI Thesaurus. Code

C117440.

The outcome of the tumor identification assessment as originally received or collected. 